\title{
An Automatic Retraining Method for Speaker Independent Hidden Markov Models
}

\author{
András Bánhalmi, Róbert Busa-Fekete, and András Kocsor \\ Research Group on Artificial Intelligence of the Hungarian Academy of Sciences \\ and of the University of Szeged, \\ H-6720 Szeged, Aradi vértanúk tere 1., Hungary \\ \{banhalmi, busarobi, kocsor\}@inf.u-szeged.hu
}

\begin{abstract}
When training speaker-independent HMM-based acoustic models, a lot of manually transcribed acoustic training data must be available from a good many different speakers. These training databases have a great variation in the pitch of the speakers, articulation and the speed of talking. In practice, the speaker-independent models are used for bootstrapping the speaker-dependent models built by speaker adaptation methods. Thus the performance of the adaptation methods is strongly influenced by the performance of the speakerindependent model and by the accuracy of the automatic segmentation which also depends on the base model. In practice, the performance of the speakerindependent models can vary a great deal on the test speakers. Here our goal is to reduce this performance variability by increasing the performance value for the speakers with low values, at the price of allowing a small drop in the highest performance values. For this purpose we propose a new method for the automatic retraining of speaker-independent HMMs.
\end{abstract}

\section{Introduction}

The probabilistic models for speech recognition are normally trained on a large amount of manually segmented data samples that contain utterances recorded from many speakers. While these speaker-independent models usually can attain a higher average accuracy on most speakers, speaker-dependent models which are trained only on the utterances of a given speaker are much more efficient in the recognition task for this specific speaker. The problem with developing speaker-dependent systems is that large amounts of speech training data for each speaker is usually unavailable. Thus in order to achieve the efficiency of the speaker dependent model, various techniques have been proposed.

Two main approaches are the transformation of the incoming feature vectors (eg. by VTLN or CMN) and the modification of the parameters of the speaker-independent acoustic models (speaker adaptation techniques). The VTLN (vocal tract length normalization) method normalizes the spectrum of the input spoken data by converting it as if all the samples had been pronounced with the same vocal tract length [1], [2], [3], while the basic CMN (cepstral mean normalization) method converts the cepstral coefficients of the input data in such a way that the samples for each speaker have the same mean value [4] [5]. The other common approach for adjusting the speaker 
Table 1. The weighted k-means method

\begin{tabular}{|c|c|}
\hline Input: & A set of M points (X) and nonnegative weights (W) \\
\hline 1 & Choose a number of k centroids from X randomly \\
\hline 2 & Repeat the next section \\
\hline 3 & $\begin{array}{l}\text { Using the given cluster centres, assign each point } \\
\text { to the cluster with the nearest center }\end{array}$ \\
\hline 4 & $\begin{array}{l}\text { Replace each cluster center by the weighted mean } \\
\text { of the points in the cluster }\end{array}$ \\
\hline 5 & $\begin{array}{l}\text { For each point, move it to another cluster, if that would } \\
\text { lower the total energy function. If a point is moved, } \\
\text { update the cluster centres of the two affected clusters. }\end{array}$ \\
\hline
\end{tabular}

independent models to better approximate the performance of speaker dependent models is speaker adaptation. In classical HMM-based systems, various speaker-adaptation techniques have been used with great success [6], [7], [8], [9], [10]. These techniques are based on the modification of the parameters of the speaker-independent system to maximize the likelihood (ML) of the adaptation data of the new speaker.

In this paper we propose a new method for improving the average accuracy after adaptation. A fundamental problem of automatic adaptation techniques is the faulty automatic segmentation, as it induces false adaptation-training data. To create a more accurate automatic segmentation, we applied an iterative method which works only on the train database, and tries to minimize the error of the segmentation. Surprisingly, not only the average accuracy of the adapted model seems to improve, but the non-adapted speaker independent model as well. The reason for this could be that by using our method the underrepresentation of certain kinds of data is reduced. And if the data representation is better, then the accuracy of the recognition and the adaptation should be better.

\section{The Retraining Procedure}

The iterative retraining method we introduce here begins with a normal Viterbi training. For each iteration the last retrained HMM is used to automatically re-segment the train database. After automatic segmentation the differences between the automatically segmented boundary positions and the manually segmented boundary positions are computed for each phoneme in the train database. The next HMM is then trained by weighting the training data according to these differences. When weighting the data, the weight of a segment is multiplied by the weight of the previous iterations. After all the weights for all the phonemes in the train database have been computed, the weighted retraining procedure begins with weighted K-Means clustering. This method is described in more detail in Table 1 The weighted mean for one cluster is computed with the following formula:

$$
\operatorname{mean}(X, W)=\frac{\sum_{i=1}^{N} W_{i} X_{i}}{\sum_{i=1}^{N} W_{i}},
$$


Table 2. The retraining method

\begin{tabular}{|c|c|}
\hline Input: & A manually segmented multi-speaker train database \\
\hline 1 & Train the initial model (M) with the normal train method \\
\hline 2 & $\mathrm{~W}=[1, \ldots, 1]$ \\
\hline 3 & Repeat the next section \\
\hline 4 & $\begin{array}{l}\text { Do automatic segmentation on the train database, } \\
\text { using the } \mathrm{M} \text { model }\end{array}$ \\
\hline 5 & $\begin{array}{l}\text { Compute the differences of the automatically and } \\
\text { manually segmented boundaries for each phoneme } \\
\text { in the train database }\end{array}$ \\
\hline 6 & $\begin{array}{l}\text { The difference value }\left(D_{i}\right) \text { for a frame }\left(x_{i}\right) \text { is computed by } \\
\text { the sum of the two boundary differences of the phoneme } \\
\text { segment containing the given frame. }\end{array}$ \\
\hline 6 & $\begin{array}{l}\text { Compute the weights }\left(W^{\prime}\right) \text { for each frame with a } \\
\text { monotone growing concave function to ensure a } \\
\text { lower sensitivity of the weighting. } \\
W^{\prime}=1+\log (D+1)\end{array}$ \\
\hline 7 & $W=W \otimes W^{\prime}$ \\
\hline 8 & Train new HMM model with weighting (using W): \\
\hline 9 & $\begin{array}{l}\text { Initialize the model using weighted k-means, } \\
\text { weighted mean, and weighted covariance }\end{array}$ \\
\hline 10 & $\begin{array}{l}\text { Train the new model }(\mathrm{M}) \text { using the weighted } \\
\text { Viterbi training method }\end{array}$ \\
\hline
\end{tabular}

where $\mathrm{X}$ contains the data points of one cluster, $\mathrm{N}$ is the number of the points in this cluster, and $\mathrm{W}$ contains their nonnegative weights. The weighted energy function of one cluster is defined as:

$$
E(X, W)=\sum_{i=1}^{N} W_{i}\left(X_{i}-\operatorname{mean}(X, W)\right)^{2},
$$

where the total weighted energy of the database is the sum of the weighted energies for each cluster.

After applying this bootstrapping procedure, the usual Viterbi training is used with the modification of computing the means and the covariances with weighting. The exact formula for the weighted mean was given previously, for the variance vector of data $\mathrm{X}$ with weights $\mathrm{W}$ (using Hadamard product):

$$
\operatorname{var}(X, W)=\frac{\sum_{i=1}^{N} W_{i}\left(X_{i}-\operatorname{mean}(X, W)\right) \otimes\left(X_{i}-\operatorname{mean}(X, W)\right)}{\sum_{i=1}^{N} W_{i}}
$$

In order to give a more precise description, the pseudo code of the iterative weighting method is given in the Table 2 below. 


\section{The Continuous Speech Recognizer and Automatic Segmentation}

The continuous speech recognizer was developed within the framework of a medical dictation project. This project was initiated by two university departments with financial support from state funds [11] [12]. Here the recognizer is based on a multi-stack Viterbi decoding and n-gram language models. All the hypotheses in a stack with the same phonetic transcriptions are merged and handled as one. Available cutting parameters are the size of the stacks, the maximal probability difference from the first hypothesis (with the highest probability), the maximal number of the new hypotheses after extension with phonemes, the maximal number of the hypotheses to be extended with phonemes, the step size of the extension with a new phoneme, the step size of the extension with a new word, along with a few less important parameters. Only the maximal probability values are computed for the states at Viterbi decoding, and some Gaussian computing speed-up techniques are applied. This continuous speech recognizer is quite effective for real-time recognition tasks with medium-sized or small-sized dictionaries.

The automatic segmentation part is incorporated in the continuous speech recognizer. Thus in this methodology the automatic segmentation is not a distinct procedure, but actually uses the same technique as the speech recognizer during testing. Thus the automatic segmentation is done using a multi-stack Viterbi decoding, and the same cutting parameters are available as mentioned above.

\section{Experiments and Results}

For training purposes speech signals from 100 speakers (26 women and 74 men) were recorded and manually segmented. The ages of the speakers were between 13 and 72. Each speaker uttered 6 long sentences (average 16 words per sentence) and 12 distinct words. The database contains about 10800 words (85300 phonemes), the total length of the speech signals is about 100 minutes. For adaptations and tests, the speech samples of 5 speakers ( 3 women and 2 men) were recorded. Each speaker uttered the same 86 sentences (613 words) for the adaptation, and 20-20 different paragraphs of medical reports [13] for tests. The total length of the recorded speech samples for adaptation was about 6 minutes and for the tests it was about 15 minutes per speaker.

For each test, the language model was the same word 3-gram model. The Hidden Markov models for 33 phonetic classes had 3 states, and each state contained 3 mixture components. All the parameters of the continuous speech recognizer were set to same value for all the test cases, and were set so that the recognition was about 1.5 times faster than real-time. Similarly, the parameters for the automatic segmentation method were set so that it was approximately 3 times faster, than real-time.

For adaptation the MAP method was used modifying only the mean values of each mixture component [14]: 


$$
\mu_{\text {new }}=\frac{N}{N+\alpha}\left(\frac{1}{N} \sum_{i=1}^{N} x_{i}\right)+\frac{\alpha}{N+\alpha} \mu_{0},
$$

The parameter $N$ represents the number of examples $\left(x_{i}\right)$ for the given mixture component, while the parameter $\alpha$ controls the speed of changing the mean of the mixture. The $\mu_{0}$ represents the initial mean value.

The performances obtained from the tests on 5 different speakers (M1, M2, W1, W2, W3) are listed in Table 3 below, while the corresponding word error rate reductions are given in Table 4 below. These results were obtained using HMMs trained after 40 iterations. The results show that the lower performance values improved by using the weighting method, while the two maximal value remained the same or fell by a few percent. The average word error rate reductions show a more significant improvement in performance after the adaptation method was applied.

Table 3. Accuracies for 5 speakers, obtained after 40 iterations. Adaptation was done with automatic segmentation using both the 'unweighted' and the 'weighted' HMMs.

\begin{tabular}{|l|c|c|c|c|c||c|}
\cline { 2 - 7 } \multicolumn{1}{c|}{} & \multicolumn{4}{c||}{ Test database } & Average \\
\cline { 2 - 7 } \multicolumn{1}{c|}{} & \multicolumn{2}{c|}{ Men } & \multicolumn{3}{c||}{ Women } & \\
\cline { 2 - 7 } \multicolumn{1}{c|}{} & M1 & M2 & W1 & W2 & W3 & \\
\hline normal & $97.57 \%$ & $98.53 \%$ & $83.42 \%$ & $95.33 \%$ & $82.99 \%$ & $91.57 \%$ \\
\hline weighted & $97.57 \%$ & $98.02 \%$ & $86.12 \%$ & $97.74 \%$ & $86.35 \%$ & $93.16 \%$ \\
\hline ad. normal & $94.43 \%$ & $95.82 \%$ & $92.42 \%$ & $98.07 \%$ & $97.60 \%$ & $95.67 \%$ \\
\hline ad. weighted & $98.43 \%$ & $96.70 \%$ & $94.52 \%$ & $98.39 \%$ & $97.67 \%$ & $97.14 \%$ \\
\hline
\end{tabular}

Table 4. Relative word error rate reductions. Here 'n.' means the normal, 'w.' means the weighted training method.

\begin{tabular}{|c||c|c|c|c|c||c|}
\hline & M1 & M2 & W1 & W2 & W3 & Average \\
\hline \hline n. $\rightarrow$ w. & $0.00 \%$ & $-34.69 \%$ & $16.28 \%$ & $51.61 \%$ & $19.75 \%$ & $18.98 \%$ \\
\hline ad. n. $\rightarrow$ ad. w. & $71.81 \%$ & $21.05 \%$ & $27.70 \%$ & $16.58 \%$ & $2.92 \%$ & $34.03 \%$ \\
\hline \hline n. $\rightarrow$ ad. n. & $-129.22 \%$ & $-184.35 \%$ & $54.28 \%$ & $58.67 \%$ & $89.89 \%$ & $48.62 \%$ \\
\hline n. $\rightarrow$ ad. w. & $35.39 \%$ & $-124.49 \%$ & $66.95 \%$ & $65.52 \%$ & $66.11 \%$ & $86.30 \%$ \\
\hline
\end{tabular}

Figure 1 shows, how the accuracy and the total sum of boundary differences vary in the first 40 iterations. It can be seen that there is some negative correlation between the difference measure, and the average accuracy. The exact correlation value is actually 0.6. In Figure 2 the accuracies on the 5 speakers vary overall together, but this tendency is less typical for speakers with higher initial performance values. The correlation values between the accuracy of the speakers are between 0.4 and 0.7 , except for the two speakers with the worst initial accuracy, whose value is 0.84 . 

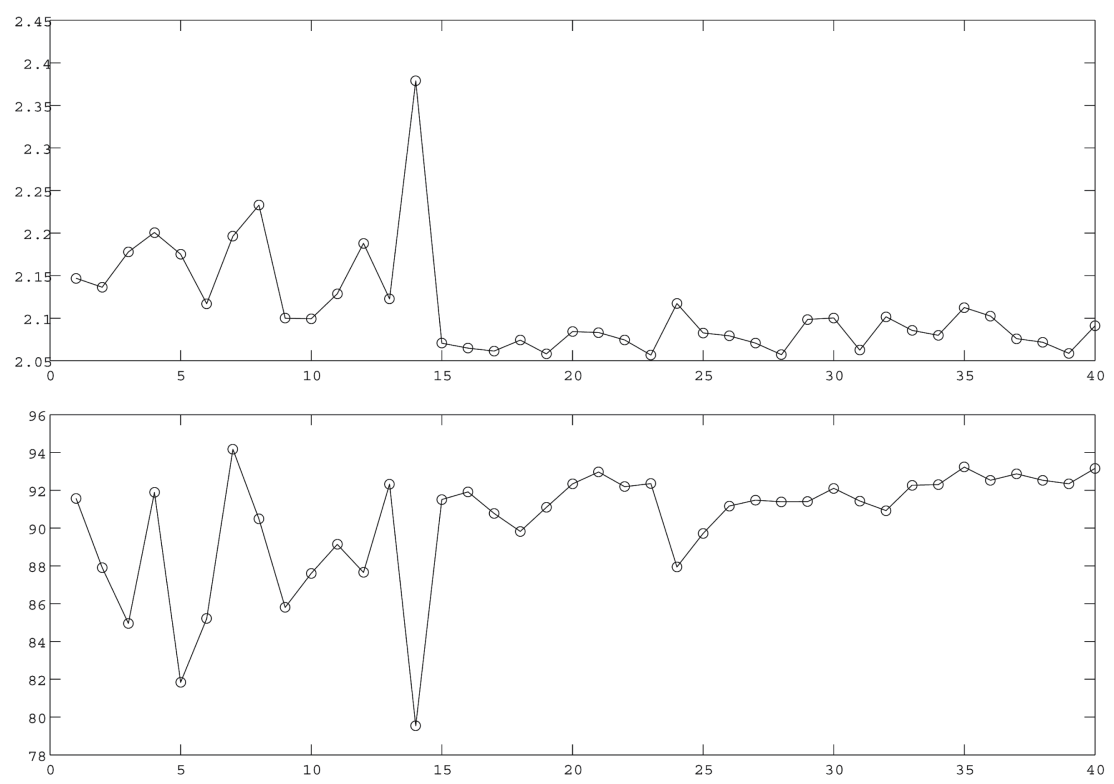

Fig. 1. The upper picture shows the total sum of boundary differences (in units of $10^{6}$ ), while the lower picture shows the average accuracy on 5 test speakers. The x-axis shows the number of training iterations performed.

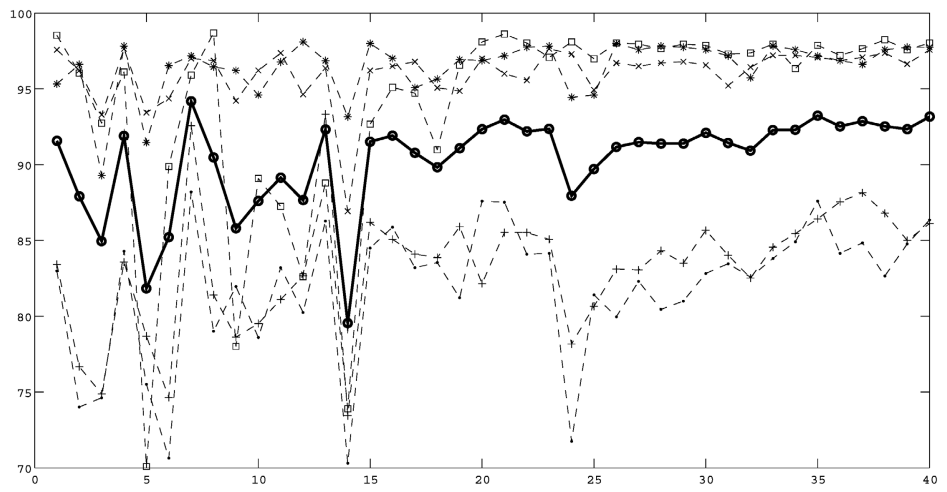

Fig. 2. The accuracies on 5 test speakers. The solid line shows the average accuracy. The x-axis shows the number of training iterations performed.

\section{Conclusions and Future Work}

The main challenge of continuous speech recognition is to improve the efficiency of the system still further. To achieve this numerous suggestions and experiments have been made. But here we tried to tackle the problem at its root that is we attempted to 
improve the recognition accuracy by modifying the training method of the base speakerindependent system. The results show that the total difference between the automatically and the manually segmented boundaries correlates with the total word error rate. By minimizing this difference we could create Hidden Markov Models with an even higher average accuracy. We constructed a simple iterative method for this minimization task, and then we got very promising results for the word error rate reduction. The increase in the accuracy is even more noticeable after speaker adaptation. The reason for this might be because the the efficiency of the adaptation methods depends much more on the performance of the base Hidden Markov Model. On the one hand there is a direct dependency: the better the initial model is, the better the adapted one will be. On the second hand there is an indirect dependency too: the better the base model is, the more accurate the automatic segmentation will be, so the adapted model will perform better. Thus one can see, that by reducing the total difference by only $4.7 \%$, the average accuracy can increase by $19 \%$, and after adaptation by $34 \%$.

We plan to continue this line of research, that is to develop methods that can raise speech recognition performance. Our next piece of work will be a new method which uses the boundary differences to initialize the Hidden Markov Models in a new way. Instead of weighting the training data, we would like to use the difference information to iteratively cluster the training data into clusters with an increasing number.

\section{References}

1. Lee, L., Rose, R.C.: Speaker normalisation using efficient frequency warping procedures. In: Proc. ICASSP96 (1996)

2. McDonough, J., Byrne, W., Luo, X.: Speaker normalization with all-pass transforms. In: Proc. ICSLP98 (1998)

3. Pitz, M., Ney, H.: Vocal tract normalization as linear transformation of mfcc. In: Proc. EuroSpeech2003 (2003)

4. Furui, S.: Cepstral analysis technique for automatic speaker verification. J. Acoust. Soc. Amer. 55, 1204-1312 (1974)

5. Kitaoka, N., Akahori, I., Nakagawa, S.: Speech recognition under noisy environments using spectral subtraction with smoothing of time direction and real-time cepstral mean normalization. In: Proceedings of the Workshop on Hands-Free Speech Communication, pp. 159-162 (2001)

6. Leggetter, C., Woodland, P.: Maximum likelihood linear regression for speaker adaptation of continuous density hmms. Computer Speech and Language 9, 171-185 (1995)

7. Gauvain, J.L., Lee, C.H.: Maximum a posteriori estimation for multivariate gaussian mixture observations of markov chains. IEEE Transactions on Speech and Audio Processing 2, 291298 (1994)

8. Sankar, A., Lee, C.: A maximum-likelihood approach to stochastic matching for robust speech recognition. IEEE Trans. on Speech and Audio Processing 3, 190-202 (1996)

9. Digalakis, V., Rtischev, D., Neumeyer, L.: Speaker adaptation using constrained reestimation of gaussian mixtures. IEEE Trans. on Speech Audio Processing, 357-366 (1995)

10. Diakoloukas, V., Digalakis, V.: Maximum-likelihood stochastic-transformation adaptation of hidden markov models. IEEE Trans. on Speech Audio Processing 2, 177-187 (1999)

11. Vicsi, K., Kocsor, A., Teleki, C., Tóth, L.: Hungarian speech database for computer-using environments in offices (in Hungarian). In: Proc. of the 2nd Hungarian Conf. on Computational Linguistics, pp. 315-318 (2004) 
12. Bánhalmi, A., Kocsor, A., Paczolay, D.: Supporting a Hungarian dictation system with novel language models (in Hungarian). In: Proc. of the 3rd Hungarian Conf. on Computational Linguistics, pp. 337-347 (2005)

13. Banhalmi, A., Paczolay, D., Toth, L., Kocsor, A.: First results of a hungarian medical dictation project. In: Proc. of IS-LTC, pp. 23-26 (2006)

14. Thelen, E.: Long term on-line speaker adaptation for large vocabulary dictation. In: Proc. of IEEE ICSPL, pp. 2139-2142. IEEE Computer Society Press, Los Alamitos (1996) 